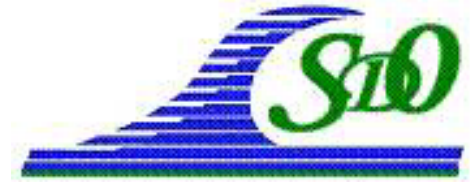

\title{
Bathymétrie littorale par télédétection : méthodes, précision et finalités
}

\section{Aurélie DEHOUCK ${ }^{1}$, Rafael ALMAR ${ }^{1}$, Yann BALOUIN ${ }^{2}$, Marion JAUD ${ }^{3}$, Christophe DELACOURT ${ }^{3}$}

1. Université de Bordeaux, UMR 5805 CNRS-EPOC, avenue des facultés, 33405 Talence cedex, France.

a.dehouck@epoc.u-bordeaux1.fr ; r.almar@epoc.u-bordeaux1.fr

2. BRGM, Service Géologique Régional du Languedoc-Roussillon,

1039 rue de Pinville, 34000 Montpellier, France.

y.balouin@brgm.fr

3. Université de Bretagne Occidentale, UMR 6538 CNRS-Domaines Océaniques,

IUEM, 29280 Plouzané, France.

marion.jaud@univ-brest.fr,christophe.delacourt@univ-brest.fr

\section{Résumé :}

Cet article fait le point sur les principales techniques d'inversion bathymétrique en zone littorale par télédétection. Ces techniques exploitent différents types de capteurs (lidar, photographie numérique, vidéo) sur des plateformes satellitaires, aéroportées ou fixes. Les mesures bathymétriques obtenues se distinguent par leur résolution spatiotemporelle, l'emprise spatiale des zones couvertes et leur précision. Ces techniques ont récemment été expérimentées sur des plages sableuses très dynamiques, évoluant rapidement sous l'action des vagues et des courants, ce qui permet d'évaluer leur pertinence vis-à-vis des échelles des différents processus d'intérêt en dynamique sédimentaire littorale.

La précision des reconstructions bathymétriques est très variable allant de 0,1 à $0,95 \mathrm{~m}$. Outre le choix de la plateforme d'observation qui détermine les échelles spatiales couvertes et la résolution planimétrique, ce sont les contraintes de mise en œuvre (météo, coût financier) et la maturité des méthodes qui conditionnent la précision de la mesure réalisée et leur positionnement vis-à-vis de la temporalité des processus et des évolutions morphologiques observés.

Mots-clés :

Bathymétrie - Cartographie - Plages - Télédétection - Imagerie - Lidar - Vidéo Satellite - Drone

\section{Introduction}

La télédétection propose aujourd'hui des outils variés et performants pour l'observation et la cartographie dynamique des systèmes sédimentaires littoraux, émergés et sousmarins, d'autant plus lorsqu'ils sont soumis à une évolution rapide, telles les plages 
sableuses, sous l'effet d'événements morphogènes récurrents (les tempêtes hivernales) ou exceptionnels. La pertinence de chacun de ces outils nécessite d'être discutée par rapport aux caractéristiques intrinsèques des objets étudiés (ex : les barres littorales), aux spatialités et temporalités de leur évolution (figure 1) :

(1) la résolution planimétrique des zones imagées doit être adaptée à la dimension des corps sédimentaires et à l'intensité de leurs déplacements ;

(2) la précision verticale de la mesure est à rapporter à l'amplitude des objets étudiés (décimétrique à métrique) et à l'ordre de grandeur des changements altimétriques ;

(3) la représentativité de la mesure et de la zone imagée cohérente avec la dimension du système étudié ;

(4) la répétitivité de la mesure doit répondre aux temporalités de la dynamique du système et à sa réactivité morphodynamique.

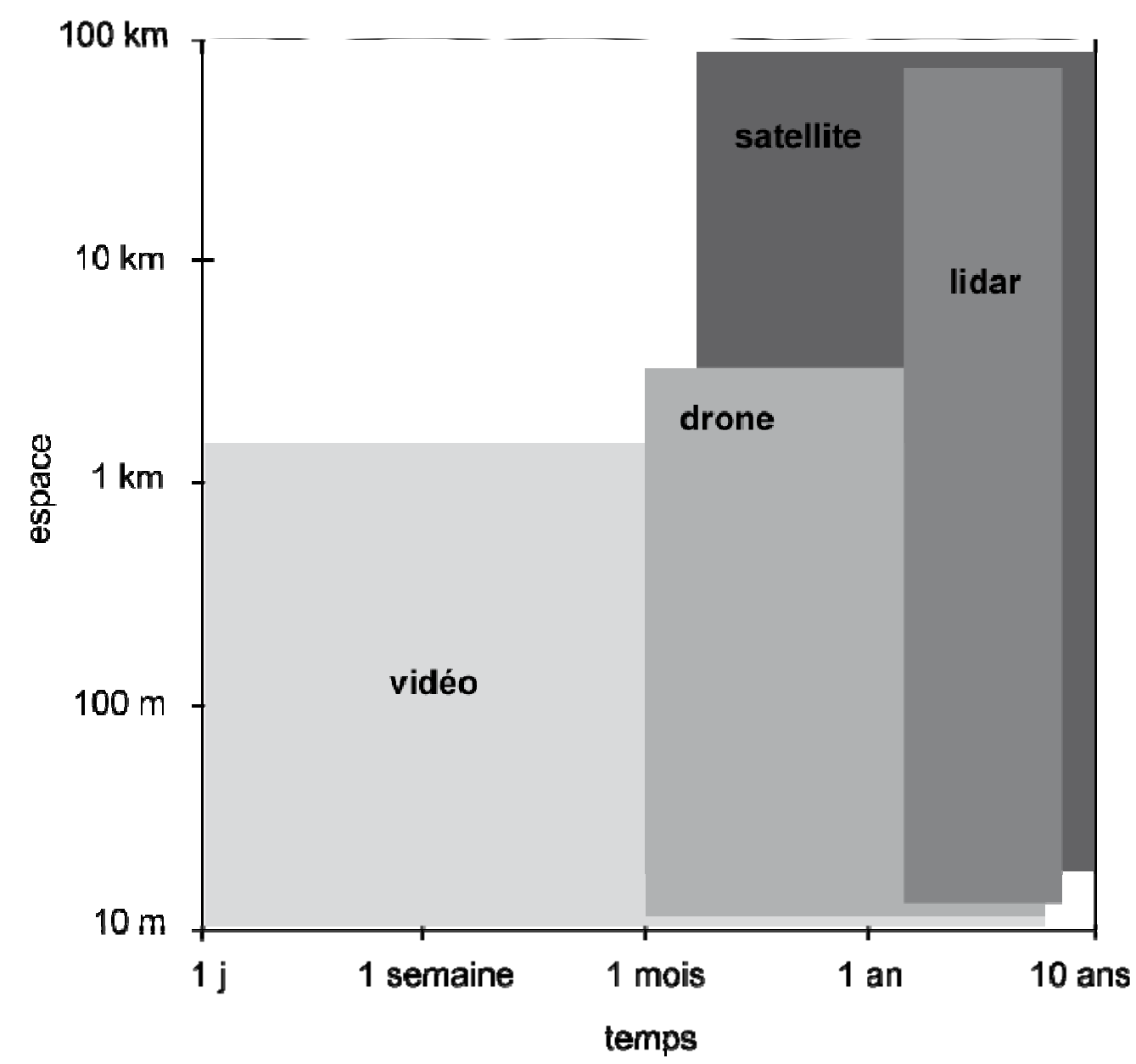

Figure 1. Positionnement des techniques de télédétection dans le domaine spatiotemporel caractéristique de l'évolution morphodynamique des littoraux sableux. 


\section{XI $I^{\text {èes }}$ Journées Nationales Génie Côtier - Génie Civil}

Les Sables d'Olonne, 22-25 juin 2010

Cet article illustre l'application de moyens de télédétection optique passive (imagerie satellitaire et vidéo terrestre) et active (lidar) à la cartographie de la bathymétrie des environnements marins sableux. Pour cela, on dispose de mesures réalisées sur trois sites ateliers sur lesquels l'une ou l'autre des techniques ont été expérimentées : les plages du Truc Vert (imagerie satellitaire) et de Biscarrosse (vidéo) sur la côte Aquitaine et la plage corse du Lido de la Marana (lidar). En outre, bien que non illustrées par des acquisitions bathymétriques sur nos sites d'étude, nous présentons le potentiel de nouvelles plateformes Drone qui se révèlent extrêmement prometteuses en termes de résolution spatiale et de coûts pour la restitution bathymétrique.

\section{Méthodes}

\subsection{Principes d'inversion bathymétrique}

\subsubsection{Radiométrie mono image}

Elle consiste en une prise de vue instantanée de la surface terrestre par réception du rayonnement solaire rétrodiffusé (télédétection optique passive) et met en œuvre les principes de la physique optique marine. L'intensité du rayonnement solaire à la surface de l'eau dépend des constituants de la colonne d'eau (concentration en particules terrigènes et biogènes), et dans les petits fonds, de la réflectivité du substrat et de la profondeur. La pénétration de la lumière dans l'eau est meilleure dans le bleu (bande spectrale B1 de Formosat-2) et le vert (bandes B2 de Formosat-2 et XS1 de SPOT) où l'effet de la présence des particules en suspension est le plus limité, le rayonnement parvenant jusqu'à une profondeur de 6-7 $\mathrm{m}$ dans les eaux moyennement turbides des Passes du Bassin d'Arcachon (LAFON et al., 2002) et littorales du Truc Vert (DEHOUCK et al., 2008).

L'inversion bathymétrique à partir des données satellitaires est réalisée en utilisant l'algorithme semi-empirique optique de LEE et al. (1998) qui s'appuie sur les propriétés optiques inhérentes (coefficients d'atténuation de la lumière descendante et montante) et apparentes du milieu (réflectance de l'eau, albedo du fond), telles que mesurées in situ par des radiomètres hyperspectraux (DEHOUCK et al., 2008).

Cette technique peut aussi être utilisée pour des acquisitions aériennes à partir de plateformes avion ou drone. L'inversion bathymétrique est alors identique à celle proposée à partir d'images acquises par des satellites. L'avantage majeur des plateformes aéroportées repose sur une meilleure résolution spatiale qui peut atteindre quelques centimètres pour les drones (LEJOT et al., 2007). En outre, l'altitude de vol étant moindre, les effets d'atténuations atmosphériques sont plus faibles. 


\subsubsection{Radiométrie continue : la vidéo}

De multiples études se sont attachées à restituer les morphologies littorales et leur évolution spatio-temporelle à partir de l'imagerie vidéo, initialement de manière qualitative. En particulier, les structures sableuses immergées sont localisables à partir des zones de dissipation d'énergie due au déferlement des vagues (LIPPMANN et HOLMAN, 1989). Plus récemment, STOCKDON \& HOLMAN (2000), HOLLAND (2001) et PLANT et al. (2008) ont développé des méthodes permettant d'estimer la bathymétrie de manière quantitative à partir de la mesure des caractéristiques locales des vagues (célérité et période), qui sont liées à la profondeur d'eau par la relation de dispersion des vagues. En se basant sur ces travaux, une méthode a été élaborée permettant une estimation plus robuste de ces caractéristiques de vague pour des conditions complexes (topographie à barre, conditions de fortes houles) (ALMAR et al., 2008). Dans un premier temps, la célérité des vagues est estimée par une intercorrélation spatio-temporelle effectuée sur les données vidéo, puis la bathymétrie est obtenue au moyen de la relation de dispersion (ici linéaire) des vagues.

\subsubsection{Mesure laser}

Le LIDAR (LIght Detection And Ranging) bathymétrique (ou hydrographique) est une technique de télédétection active (émission et réception). Le système permet les levés topographique et bathymétrique simultanés. Le laser topographique émet un rayon de $1064 \mathrm{~nm}$ (proche infra rouge) qui permet la détection de la surface de l'eau et le laser bathymétrique un rayon à $532 \mathrm{~nm}$ (bleu-vert) qui pénètre dans l'eau. Le système opère à une fréquence de $4000 \mathrm{~Hz}$. Le rayonnement lumineux émis par le capteur pénètre dans la colonne d'eau, plus ou moins profondément selon sa clarté. Le signal retour, réfléchi par le fond, est réceptionné et analysé dans le domaine temporel pour en déduire la distance séparant la plateforme lidar et la surface impactée et ainsi son élévation. Le système de détection multi-pixel d'Hawk Eye II permet d'obtenir des densités de l'ordre de 0,45 points $/ \mathrm{m}^{2}$ (1 point tous les $\left.1,5 \mathrm{~m}\right)$ en bathymétrie et supérieures à 6 points $/ \mathrm{m}^{2}(1$ point tous les $0,4 \mathrm{~m}$ ) en topographie.

\subsection{Plateformes de déploiement}

\subsubsection{Plateformes fixes}

Utilisée à partir d'une base fixe positionnée sur la dune ou une falaise, la mesure vidéo est caractérisée par une large couverture temporelle (de la seconde à l'année) et par une très haute résolution spatiale (pixel de quelques centimètres sur la plage intertidale au plus près des caméras, de 5 à $10 \mathrm{~m}$ au large). L'emprise de la zone imagée est déterminée par le nombre de caméras déployées, de l'ordre de 1 à $2 \mathrm{~km}$ de linéaire côtier et jusqu'à $1 \mathrm{~km}$ vers le large. 


\section{XI ${ }^{\text {èmes }}$ Journées Nationales Génie Côtier - Génie Civil \\ Les Sables d'Olonne, 22-25 juin 2010}

Les principaux avantages sont: (1) la possibilité nouvelle d'une observation continue de la dynamique littorale, permettant de ne manquer aucun évènement scientifiquement intéressant (tempête), de le suivre à haute fréquence et de capter l'évolution rapide des structures sableuses impactées ; (2) la pérennité de la mesure qui autorise la génération de bases de données long terme et d'études statistiques; (3) la très haute résolution spatiale qui permet de caractériser finement la morphologie des structures sableuses ; (4) la dualité hydrodynamique et morphologique de la mesure, unique parmi les outils d'observation littorale, permettant la compréhension des processus hydrologiques à l'origine des changements morphologiques observés.

\subsubsection{Plateformes aéroportées}

Diverses plateformes aéroportées peuvent intégrer des capteurs optiques passifs ou actifs dans le but de cartographier la frange littorale sous-marine. En raison de la masse importante des capteurs, les levés LIDAR sont réalisés en hélicoptère ou en avion selon les caractéristiques de la zone à lever et la résolution attendue. Le vol s'effectue à des altitudes généralement comprises entre $200 \mathrm{~m}$ et $500 \mathrm{~m}$. Toute plateforme aéroportée est constituée d'un système de positionnement (GPS cinématique en temps réel) couplé à une centrale d'attitude et à un magnétomètre.

Depuis quelques années, les Drones sont utilisés dans différents contextes d'observation de la Terre (agriculture (SUGIURA et al., 2005), rivières (LEJOT et al., 2007), glissements de terrain (CASSON et al., 2005)) et peuvent avantageusement remplacer les plateformes aériennes conventionnelles lorsque les capteurs sont relativement légers (une dizaine de kilogrammes). Les drônes offrent, pour un coût réduit, une grande souplesse d'utilisation : programmation de mission avec des délais réduits (moins d'une journée), facilité de mise en œuvre à proximité immédiate de la zone d'intérêt, maniabilité, vol à faible altitude (n'étant pas soumis à la même réglementation que les avions ou hélicoptères). Ils se prêtent donc parfaitement à des suivis de zones de taille « réduite » et requérant une très haute résolution spatiale (centimétrique). La fréquence des survols (souvent mensuelle à annuelle) est adaptée en fonction du phénomène à observer. Un drone à vocation littorale a été développé (DELACOURT et al., 2009). C'est un hélicoptère acrobatique thermique Vario. Pouvant supporter une charge de $6 \mathrm{~kg}$ et voler avec des vents supérieurs à 10 nœuds, il ne permet pas d'embarquer un LIDAR mais est équipé d'un auto-pilote et de capteurs optiques (appareil photographique réflex de grande qualité ou caméra thermique) en vue de suivis photogrammétriques ou radiométriques. Ce système a été testé pour restituer la bathymétrie d'une Lone (LEJOT et al., 2007) avec une précision de $10 \mathrm{~cm}$ pour des profondeurs allant jusqu'à $2,4 \mathrm{~m}$

\subsubsection{Plateformes satellitaires}

La télédétection spatiale met en œuvre des capteurs optiques passifs installés à bord de satellites d'observation de la Terre (altitude 700 à $800 \mathrm{~km}$ ). Ils fournissent des prises de 
vue de haute résolution spatiale (décamétrique) à très haute résolution spatiale (submétrique) bien adaptés à l'observation des morphologies littorales. Ce sont des produits intéressants pour cartographier la frange littorale à l'échelle régionale étant donné l'emprise spatiale des zones couvertes (20 à $60 \mathrm{~km}$ ). Cependant, nombre de ces capteurs ont une fréquence de passage hebdomadaire voire pluri-hebdomadaire qui conjuguée à de mauvaises conditions météorologiques (couverture nuageuse) peuvent rendent extrêmement contraignant ce type d'observation. C'est pourquoi, la télédétection spatiale est adaptée à l'observation des systèmes littoraux au mieux à l'échelle saisonnière. Toutefois, de plus en plus de capteurs sont amenés à couvrir quotidiennement une même zone du globe (aujourd'hui Formosat-2, demain la constellation Pléiades) permettant une meilleure réactivité lors d'événements climatiques exceptionnels.

\section{Résultats}

\subsection{Imagerie satellitaire}

Cette méthode a été testée sur la plage du Truc Vert (DEHOUCK et al., 2009) à partir de la bande spectrale B1 d'une image satellite Formosat-2 (figure 2a). La précision des reconstitutions bathymétriques (figure $2 b$ ), évaluée à partir des levés quasi-simultanés du SHOM (160000 sondes pour 11700 pixels), atteint $0,42 \mathrm{~m}$ (erreur relative 11\%) pour des hauteurs d'eau de 0 à $6 \mathrm{~m}$ (figure 2c). La performance de l'algorithme se révèle satisfaisante lorsqu'il est optimisé avec un coefficient d'atténuation de la lumière dans l'eau variable sur la zone d'intérêt. Pour exemple, malgré la qualité de l'image exploitée, une défaillance de l'algorithme a été initialement constatée au niveau de la crête de la barre sous-marine (surestimation des profondeurs jusqu'à 1,5 m). Là, une remise en suspension très localisée des sédiments peut avoir lieu sous l'action des vagues rendant les eaux sensiblement plus turbides.

\subsection{Imagerie vidéo}

Cette nouvelle méthode a pu être validée en utilisant les données des campagnes de terrain PreECORS 2007 et ECORS 2008 sur la côte aquitaine. Les données vidéo ont été comparées aux mesures in situ des vagues et à des bathymétries de grande emprise spatiale du SHOM. La comparaison des données hydrodynamiques a montré que l'erreur sur la mesure vidéo des caractéristiques des vagues est inférieure à $10 \%$ (soit environ $<0,2 \mathrm{~m} / \mathrm{s}$ et $0,5 \mathrm{~s}$, respectivement pour la célérité et la période des vagues) (ALMAR et al., 2008). La comparaison entre la topographie vidéo estimée et celle mesurée (figure 3) montre que l'estimation vidéo représente assez correctement la géométrie des structures pour des profondeurs allant jusqu'à $10 \mathrm{~m}$. L'amplitude des barres sableuses reste cependant mal appréciée, la profondeur de la barre d'avant-plage $(\mathrm{X}=650 \mathrm{~m})$ étant sur-estimée et une petite structure absente de la topographie in situ 


\section{XI $I^{\text {èes }}$ Journées Nationales Génie Côtier - Génie Civil}

Les Sables d'Olonne, 22-25 juin 2010

apparaissant malgré tout dans la topographie vidéo $(X=480 \mathrm{~m})$. Il en résulte une erreur RMS très importante $(0,95 \mathrm{~m})$ du fait d'une qualité de la mesure très dégradée au niveau des barres immergées avec des erreurs maximales localisées là où les effets nonlinéaires du au déferlement des vagues sont prépondérants. Ceci suggère une mauvaise représentation des vagues par la théorie linéaire à ces endroits précis. Aussi, une amélioration envisagée est l'application d'un modèle de vague plus réaliste, non-linéaire (CATALAN et al., 2008), pour réaliser l'inversion bathymétrique. L'erreur sur un profil moyenné le long du littoral ( $800 \mathrm{~m}$ de linéaire côtier) obtenu à partir de quatre jours de données vidéo se réduit à $0,3 \mathrm{~m}$; les profils topographiques terrain et vidéo présentant alors un meilleur accord quant à la localisation et l'amplitude verticale des barres sableuses littorales (figure non présentée). Cette méthode permet donc de quantifier l'évolution de la morphologie littorale à haute fréquence (jour), à l'échelle de la plage $\left(1 \times 1 \mathrm{~km}^{2}\right)$. La précision reste toutefois à améliorer pour une utilisation opérationnelle.
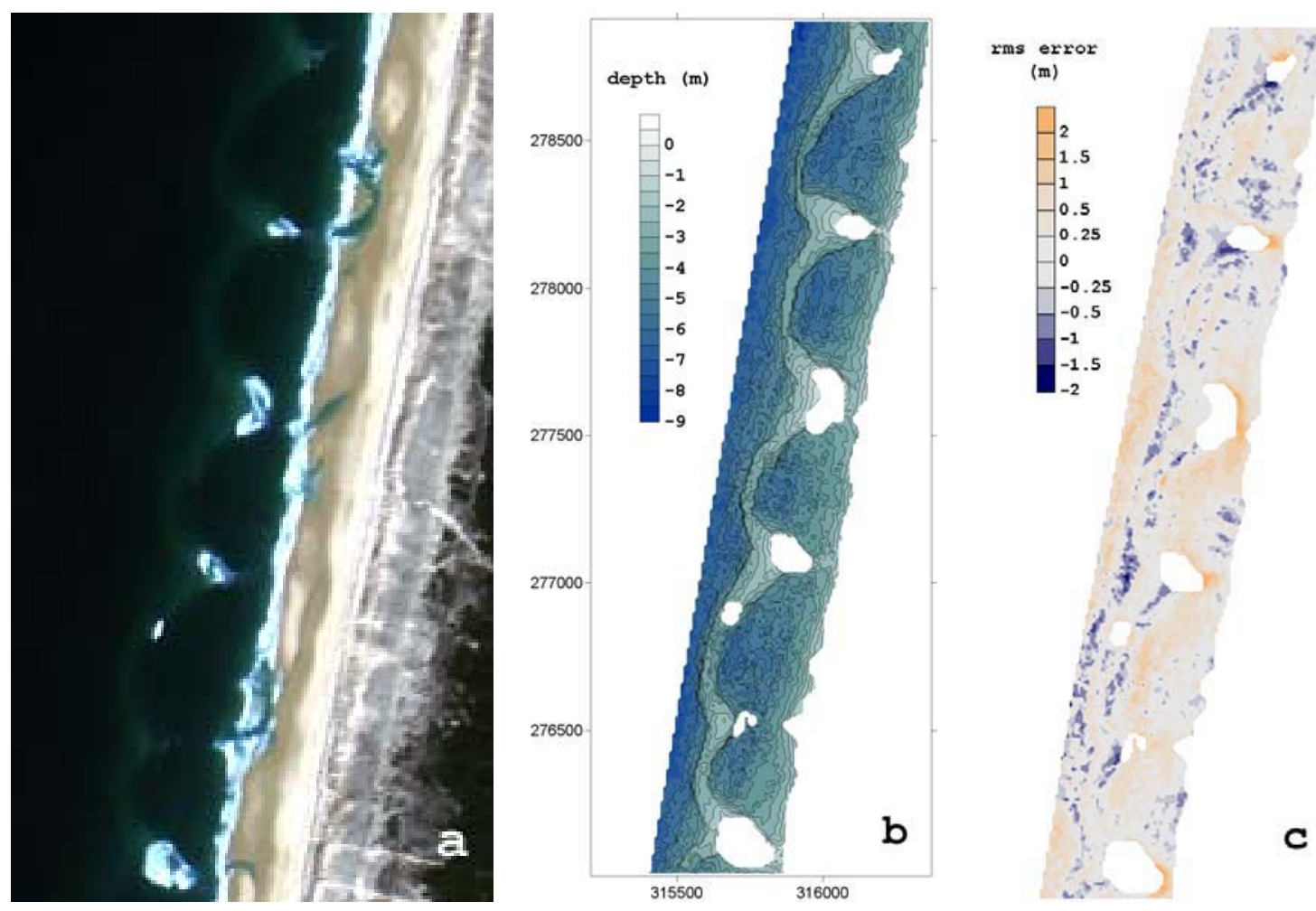

Figure 2. (a) Extrait de la scène Formosat-2 du 10/2/08 sur la zone du Truc Vert (C) Kalideos Littoral CNES) ; (b) spatio-carte bathymétrique de la plage sous-marine ; (c) erreur rms $z$ satellite $-z$ in situ $(m)$. 

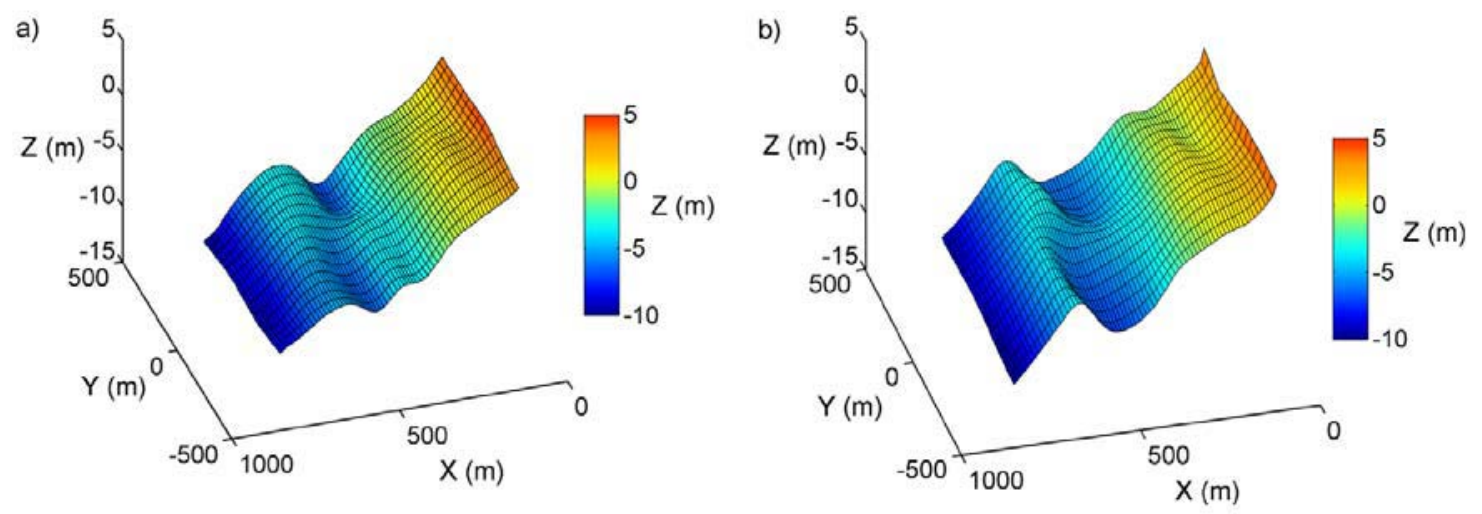

Figure 3. Topographies littorales obtenues à partir de (a) la méthode vidéo et (b) du relevé in-situ réalisé par le SHOM (modifié à partir de ALMAR, 2009).

\subsection{Levé Lidar}

La précision des données obtenues par le LIDAR bathymétrique a été évaluée lors d'une campagne test sur le Lido de la Marana en Corse avec un capteur HAWK EYE II. Une comparaison entre les données LIDAR et celles obtenues avec un sondeur monofaisceau (figure 4) met en évidence la très bonne précision altimétrique de la mesure LIDAR (10,4 cm et $70 \%$ des 1200 points comparés ayant un écart inférieur à $5 \mathrm{~cm})$. La densité moyenne constatée lors de ce levé est de l'ordre de 0,6 points $/ \mathrm{m}^{2}$ (résolution métrique), un recouvrement de $50 \%$ entre deux lignes de vol successives étant nécessaire pour obtenir une densité plus homogène et importante.

L'interprétation du signal de retour du LIDAR bathymétrique ne permet pas de caractériser le fond dans les très faibles profondeurs (cotes de 0 à $-30 \mathrm{~cm}$ avec un léger clapot), en raison d'une superposition des signaux correspondant à la surface de l'eau et au fond. Par ailleurs, les zones d'embouchures, qui présentaient des turbidités un peu plus importantes, n'ont pu être levées de manière satisfaisante. Sur le littoral corse, le levé, réalisé en mars 2006 dans des conditions peu optimales, a toutefois permis d'atteindre des profondeurs de l'ordre de $25 \mathrm{~m}$ (les profondeurs plus importantes n'ayant pas été investiguées). 

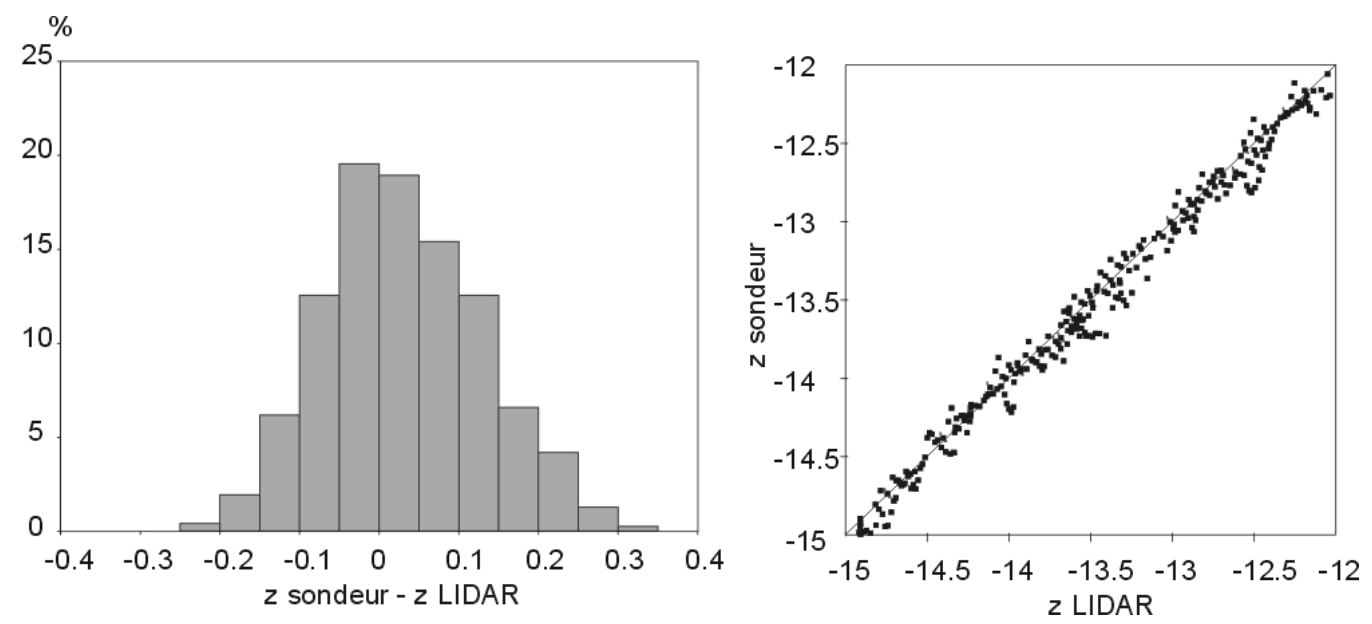

Figure 4. Evaluation altimétrique du levé Lidar. (a) spectre de fréquence de la différence altimétrique ( $z$ en $m$ ) entre les deux techniques ; (b) nuage de points de l'élévation obtenue par sondeur et lidar (d'après BALOUIN \& HEURTEFEUX, 2007).

\section{Discussion}

La résolution spatiale, sub-métrique, est pertinente pour chacun des outils, notamment avec l'arrivée des nouveaux capteurs satellitaires de très haute résolution. Les contraintes techniques à la mise en œuvre de ces outils sont sensiblement les mêmes, afférentes aux conditions météorologiques (couverture nuageuse, vent, pluie) et d'agitation (turbidité, déferlement ou zone de surf saturée). Plusieurs des technologies présentées ici (imagerie satellitaire, levé lidar) tirent leur performance à restituer la bathymétrie des petits fonds (tableau 1) de la transparence du milieu, ce qui les rend inopérables dans des environnements turbides (embouchures, mangroves), voire même sur les plages ouvertes lorsque la remise en suspension des sables est excessive. Seule l'imagerie vidéo (et l'imagerie SAR non abordée dans cet article) évite cet écueil en s'appuyant sur les propriétés physiques de la propagation des vagues, mais non sans difficulté puisque la non-linéarité des processus physiques en zone de surf complexifie l'algorithmie et pénalise la performance actuelle de la méthode d'inversion bathymétrique à partir de l'imagerie vidéo.

Jusqu'à récemment, la dynamique court-terme (jour-semaine) des littoraux sableux, pourtant supposée importante (environ un tiers de la variabilité totale; ALMAR, 2009) restait méconnue, de par le manque d'outil d'observation adapté. L'utilisation de l'imagerie vidéo permet de palier à cette lacune, les caractéristiques spatio-temporelles intermédiaires de cette méthode faisant le lien entre la petite et la grande échelle littorale. L'imagerie vidéo apparait alors comme complémentaire à d'autres techniques comme le lidar, plus précise mais utilisée de manière ponctuelle ou l'imagerie satellitale adaptée aux grandes échelles spatiales. En effet, bien qu'étant la technologie la plus opérationnelle, le coût financier reste le frein majeur à l'exploitation du lidar en domaine littoral (tableau 1), coût qui pénalise la répétition des observations pourtant 
essentielle à la quantification de l'évolution des systèmes littoraux. Ainsi, il n'existe pas d'unicité des technologies pour intégrer les contraintes et la diversité des échelles caractérisant la dynamique des corps sédimentaires littoraux et leurs évolutions.

Tableau 1. Performance de la restitution de la bathymétrie à partir des différentes techniques de télédétection en termes de résolution, de précision altimétrique et de coût.

\begin{tabular}{|c|c|c|c|c|}
\hline & Produits & $\begin{array}{c}\text { Résolution } \\
\text { planimétrique } \\
\text { (m) }\end{array}$ & $\begin{array}{c}\text { Précision } \\
\text { altimétrique } \\
\text { (m) }\end{array}$ & $\begin{array}{c}\text { Coût financier } \\
\text { (€/km²/levé) } \\
\text { * investissement } \\
\text { de départ (€) }\end{array}$ \\
\hline Sondeur hydrographique & SHOM & $0,5-5$ & $0,1-0,2$ & $2500-5000$ \\
\hline Vidéo & EPOC & $0,05-10$ & 0,95 & $20000^{*}$ \\
\hline Lidar & Hawk Eye II & 1,5 & 0,10 & $1500-6000$ \\
\hline Satellite & Formosat-2 & 8 & 0,42 & $130-250$ \\
\hline
\end{tabular}

\section{Conclusion}

Cet article évalue le potentiel de trois outils de télédétection (1) l'imagerie vidéo implantée sur site, (2) le lidar aéroporté et (3) l'imagerie satellitale optique pour cartographier la bathymétrie des littoraux sableux. Leur performance altimétrique est très hétérogène, décamétrique à métrique, conditionnée par leur degré de maturité. Compte tenu des contraintes techniques, méthodologiques et financières, il n'y a pas d'outil d'observation unique mais une gamme complémentaire couvrant le spectre des spatialités et temporalités caractéristiques des dynamiques sédimentaires littorales.

\section{Remerciements}

Les auteurs souhaitent remercier le CNES et la DGA pour le financement des travaux d'A. Dehouck et de R. Almar, la mise à disposition des images satellite dans le cadre de la base Kalideos Littoral (CNES), le financement des campagnes pré ECORS 2007 et ECORS 2008 (BRGM, SHOM-DGA) ayant permis la collecte des données in situ de calibration et validation des méthodes d'inversion basées sur la vidéo et l'imagerie satellitaire.

\section{Références bibliographiques}

ALMAR R., BONNETON P., SENECHAL N., ROELVINK D. (2008). Video observations of beach cusp morphodynamics. Proceedings of the ICCE2008 Conference, Hamburg.

ALMAR R. (2009), Morphodynamique littorale haute fréquence par imagerie vidéo. Thèse, Université de Bordeaux, $233 \mathrm{p}$. 
BALOUIN Y., HEURTEFEUX H. (2007). Utilisation de la technologie du lidar bathymétrique pour le suivi du littoral: retour d'expériences sur le littoral méditerranéen. Revue Française de Photogrammétrie et de Télédétection, $\mathrm{n}^{\circ} 186$, pp 74-80.

CASSON B., DELACOURT C., ALLEMAND P. (2005). Contribution of multitemporal remote sensing images to characterize landslide slip surface - Application to the La Clapière landslide (France). Natural Hazards and Earth System Science, 5, pp 425-437.

CATALAN P.A., HALLER M.C. (2008). Remote sensing of breaking wave phase speeds with application to non-linear depth inversions. Coastal Engineering, vol. 55, pp 93-111. doi:10.1016/j.coastaleng.2007.09.010

DEHOUCK A., MARTINY N., FROIDEFOND J.M., SENECHAL N., LAFON V., BUJAN S. (2008). In-water reflectance spectra measured on-board a jet-ski across a complex nearshore zone of case-2 waters during the ECORS experiment. Proceedings of Ocean Optics XIX, Barga, 8 p.

DEHOUCK A., MARTINY N., FROIDEFOND J.M., SENECHAL N., BUJAN S. (2009). New outcomes from spatial remote sensing during the ECORS experiment : towards validation of ocean color products and large-scale bathymetry mapping in a coastal zone. Journal of Coastal Research, SI 56(2), pp 1756-1760.

DELACOURT C., ALLEMAND P., JAUD M., GRANDJEAN P., DESCHAMPS A., AMMANN J., CUQ V., SUANEZ S. (2009). DRELIO: an unmanned helicopter for imaging coastal areas. Journal of Coastal Research, SI 56(2), pp 1489-1493.

HOLLAND K.T. (2001). Application of the linear dispersion relation with respect to depth inversion and remotely sensed imagery. IEEE Transactions on Geoscience and Remote Sensing, vol. 39, pp 2060-2072. doi:10.1109/36.951097

LAFON V., FROIDEFOND J.M., LAHET F., CASTAING P. (2002). SPOT shallow water bathymetry of a moderately turbid tidal inlet based on field measurements. Remote Sensing of Environment, vol. 81, pp 136-148. doi:10.1016/S0034-4257(01)00340-6 LEE Z., CARDER K.L., MOBLEY C.D., STEWARD R.G., PATCH J.S. (1998). Hyperspectral remote sensing for shallow waters. I. a semianalytical model. Applied Optics, vol. 37(27), pp 6329-6338. doi:10.1364/AO.37.006329

LEJOT J., DELACOURT C., PIEGAY H, FOURNIER T, TREMELO M.L., ALLEMAND P. (2007). Very high spatial resolution imagery for channel bathymetry and topography from an unmanned mapping controlled plateform. Earth Surface Processes and Landforms, vol. 32 (11), pp 1705-1725. doi:10.1002/esp.1595

LIPPMANN T.C., HOLMAN R.A. (1989). Quantification of sand bar morphology: a video technique based on wave dissipation. Journal of Geophysical Research, vol. 94 (C1), pp 995-1011. doi:10.1029/JC094iC01p00995 
Thème 3 - Instrumentation, mesures, imagerie et télédétection

PLANT N.G., HOLLAND K.T., HALLER M.C. (2008). Ocean wavenumber estimation from wave-resolving time series imagery. IEEE Transactions on Geoscience and Remote Sensing, vol. 46, pp 2644-2658. doi:10.1109/TGRS.2008.919821

STOCKDON H.F., HOLMAN R.A. (2000). Estimation of wave phase speed and nearshore bathymetry from video imagery. Journal of Geophysical Research, vol. 105, pp 15-22. doi:10.1029/1999JC000124

SUGIURA R., NOGUCHI N., ISHII K. (2005). Remote-sensing technology for vegetation monitoring using an unmanned helicopter. Biosystems engineering, 90 (4), pp 369-379. doi:10.1016/j.biosystemseng.2004.12.011 